Meta

Journal des tradlucteurs

Translators' Journal

\title{
Sondage sur la traductrice québécoise
}

\section{Mireille Boucher et Johanne Thomas}

Volume 27, numéro 4, décembre 1982

URI : https://id.erudit.org/iderudit/002107ar

DOI : https://doi.org/10.7202/002107ar

Aller au sommaire du numéro

Éditeur(s)

Les Presses de l'Université de Montréal

ISSN

0026-0452 (imprimé)

1492-1421 (numérique)

Découvrir la revue

Citer cette note

Boucher, M. \& Thomas, J. (1982). Sondage sur la traductrice québécoise. Meta, 27(4), 450-453. https://doi.org/10.7202/002107ar d'utilisation que vous pouvez consulter en ligne.

https://apropos.erudit.org/fr/usagers/politique-dutilisation/ 
BLOC-NOTES

\section{SONDAGE SUR LA TRADUCTRICE QUÉBÉCOISE}

\section{Introduction}

Qui est la traductrice québécoise? D'où vientelle? Quels sont ses intérêts? Est-elle satisfaite de sa profession? Telles sont les questions auxquelles nous cherchions une réponse au moment d'entreprendre notre enquête. C'est pourquoi nous avons divisé notre questionnaire en six parties: renseignements personnels, scolarité, renseignements professionnels, revenu, loisirs et degré de satisfaction au travail. Parmi les points qui nous intéressaient, les avantages et les inconvénients du travail à la pige par rapport au travail de non-pigiste figuraient au premier plan. Nous supposions en effet que les femmes pigistes avaient choisi de travailler de cette façon parce qu'elles avaient des enfants à la maison. Nous nous sommes aussi demandé si, dans un milieu de travail mixte où les femmes sont majoritaires, l'échantillonnage cadres féminins/cadres masculins était respecté

Pour établir notre échantillonnage, nous avons utilisé le répertoire de la Société des traducteurs du Québec (STQ) qui contient les noms de 1500 traducteurs-membres, dont les deux tiers sont des femmes. Afin d'obtenir un échantillon de cent sujets, représentant environ $10 \%$ de la population des traductrices, nous avons sélectionné 136 noms de façon aléatoire. Nous avons réussi à rejoindre, par téléphone, 105 candidates : 14 ont refusé de répondre au questionnaire et 91 ont accepté de le faire, ce qui représente un excellent taux de réponse de $86,6 \%$.

\section{Analyse des données}

\section{Renseignements personnels}

Analysons tout d'abord les résultats se rapportant aux renseignements personnels.

- Premièrement, il ressort que les traductrices sont surtout des femmes jeunes, 60,3\% ayant moins de 40 ans. On compte cependant une proportion importante de personnes dans la force de l'âge, $23 \%$ ayant entre 40 et 49 ans. Certaines traductrices continuent à travailler même après 65 ans (voir tableau 1)

- Deuxièmement, près des trois quarts des répondantes sont nées au Québec, mais $24,2 \%$ sont originaires d'un pays étranger.

- Troisièmement, les traductrices sont issues, en général, d'un milieu socio-économique favorisé. Les résultats indiquent en effet que d'une part, le père exerce, dans la majorité des cas, une profession libérale $(\mathbf{4 4 \%})$ et a plus de 16 ans de scolarité (39\%) et que, d'autre part, la mère ne travaille pas à l'extérieur dans $64 \%$ des cas, et a complété des études secondaires dans la proportion de $60 \%$.

- Quatrièmement, comme nous l'avions prévu, le français est la langue maternelle de $88 \%$ des répondantes, et l'anglais la langue seconde dans $87 \%$ des cas. Nous nous sommes également intéressées au moyen et à l'âge d'acquisition de la langue seconde. Avant l'âge de cinq ans, $23 \%$ des traductrices qui ont participé au sondage parlaient déjà une deuxième langue, l'ayant apprise ou à la maison ou avec des amis. Nous constatons que la majorité (39) ont appris leur langue seconde entre six et douze ans, et que dans $79 \%$ des cas, le lieu d'apprentissage était l'école. Pour celles qui l'ont apprise entre treize et vingt ans (32\%), c'est encore l'école qui est le lien d'acquisition privilégié. Néanmoins, l'apprentissage avec des amis occupe aussi une place importante (voir tableaux 2 et 3).

- Cinquièmement, en ce qui a trait à l'état matrimonial des répondantes, la moitié sont présentement mariées, environ $13 \%$ séparées ou divorcées et $4 \%$ veuves. Il est à noter que si $33 \%$ des participantes ne sont pas mariées et se déclarent célibataires, elles vivent néanmoins avec un concubin dans le tiers des cas. Par contre, au moins le quart des répondantes vivent seules. 
- Finalement, nous constatons que $40 \%$ des participantes ont des enfants à la maison et que celles-ci se retrouvent dans les catégories de participantes qui sont mariées ou qui l'ont déjà été. Par ailleurs, les familles monoparentales sont quasi inexistantes. Si une traductrice sur deux, ou presque, a des enfants, les familles sont toutefois peu nombreuses puisque $78 \%$ d'entre elles ne comptent pas plus de deux enfants.

\section{Scolarité}

La deuxième partie de notre questionnaire portait sur la scolarité. La presque totalité des traductrices ont une formation universitaire $(96,7 \%)$. De ce nombre, $55 \%$ ont un baccalauréat spécialisé en traduction et $30,7 \%$ en arts et lettres (spécialement les traductrices plus âgées). Un peu plus du tiers des traductrices ont une maîtrise mais, fait important à noter, la plupart se sont spécialisées dans le domaine des arts et des lettres plutôt qu'en traduction. Nous avons pu également remarquer que les traductrices n'ont pas changé d'orientation tout au long de leurs études, les seules modifications consistant souvent à passer de la traduction aux lettres ou inversement.

\section{Renseignements professionnels}

Examinons maintenant les données relatives aux renseignements professionnels. L'information obtenue sur la vie professionnelle des traductrices nous a permis d'établir une comparaison entre les pigistes et les non-pigistes. Notre échantillonnage a été élaboré de façon à ce que les deux groupes soient représentés également, mais le nombre de pigistes qui ont répondu est demeuré inférieur à nos prévisions. Les résultats du sondage démontrent qu'un peu plus du tiers des participantes actives sont pigistes, ce qui nous permet tout de même d'établir un parallèle entre les deux groupes. Analysons tout d'abord la situation des non-pigistes.

La première constatation est que la grande majorité d'entre elles $(79 \%)$ travaille pour l'entreprise privée, tandis qu'environ un dixième travaille à la fonction publique et un autre dixième pour des cabinets de traduction.

Deuxièmement, $90 \%$ des non-pigistes travaillent à temps plein, ce qui représente une semaine de travail de 37 heures pour plus du tiers d'entre elles. Le sondage démontre également que $25 \%$ des participantes travaillent entre 31 et 35 heures par semaine. Par contre, il peut paraître étonnant de noter qu'encore aujourd'hui près de $10 \%$ travaillent 40 heures par semaine.

Nous nous sommes également penchées sur la représentativité des traductrices parmi les cadres. Nous avons appris que dans les bureaux de traduction $74 \%$ des traducteurs sont des fem- mes et $26 \%$ des hommes. Nous retrouvons sensiblement la même proportion en analysant les pourcentages de traducteurs-cadres, $71 \%$ étant des femmes et $29 \%$ des hommes. Donc contrairement à ce qui se passe dans plusieurs professions, les femmes sont convenablement représentées parmi les cadres. Serait-ce parce qu'elles sont majoritaires dans la profession (voir tableau 4)?

Penchons-nous maintenant sur la situation des pigistes, qui représentent $37 \%$ de nos répondantes. La plupart d'entre elles travaillent à temps partiel $(81 \%)$. Contrairement à notre hypothèse de départ, le sondage révèle que, dans $\mathbf{5 0 \%}$ des cas, les pigistes se sont tournées vers cette forme de travail par goût et non pas parce qu'elles avaient ou voulaient des enfants. Il est quand même important de noter qu'un tiers d'entre elles ont donné comme raison de ce choix la présence d'enfants à la maison.

Nous avons ensuite tenté de déterminer les avantages et les inconvénients du travail à la pige. La grande majorité des répondantes ont indiqué qu'il était avantageux de pouvoir choisir sa clientèle, ses domaines de traduction et ses heures de travail, et qu'elles aimaient bien travailler seules. Par contre, l'absence de bénéfices marginaux et l'irrégularité du travail présentent pour elles des inconvénients.

Après avoir brossé un tableau distinctif entre pigistes et non-pigistes, nous avons voulu savoir dans quels domaines les unes et les autres se spécialisent. Près des trois quarts (70\%) travaillent effectivement dans des domaines spécialisés, alors que $29 \%$ font de la traduction générale. Le plus fort pourcentage $(31 \%)$ travaillent dans le domaine de la traduction commerciale, qui comprend la traduction administrative, financière, etc. En deuxième lieu, 16\% font de la traduction technique (voir tableau 5). On remarque qu'aucune des traductrices comprises dans notre échantillon ne fait de traduction littéraire, même parmi les pigistes. Nous avons également appris que $60,5 \%$ des répondantes ont exercé des fonctions différentes auparavant: secrétaires $(33 \%)$, enseignantes $(28 \%)$. Ces résultats s'expliquent facilement. En effet, la plupart des anciennes secrétaires nous ont dit qu'elles avaient décidé de devenir traductrices après avoir été appelées à traduire occasionnellement; elles ont donc suivi des cours de traduction le soir. Quant aux enseignantes, elles manifestent peut-être une tendance, générale il y a une génération. L'École normale étant un des rares débouchés réservés aux femmes qui voulaient travailler, plusieurs d'entre elles sont devenues enseignantes par la force des choses et ont changé de carrière par la suite lorsque la situation eut évolué. Enfin les réponses relatives aux années d'expérience en traduction ont révélé que $40 \%$ des répondantes ont 5 années d'expérience ou moins et que près de $30 \%$ 
d'entre elles en ont entre 6 et 10 . A première vue, ces pourcentages semblent élevés, mais ils s'expliquent si on tient compte des résultats obtenus précédemment à l'effet que la traduction soit une deuxième carrière pour $60 \%$ des répondantes. Il est donc logique qu'en général le nombre d'années d'expérience moyen soit relativement bas, si on le compare à celui des autres professions comme la médecine, le génie, le droit, où la moyenne est de 20 ans car les praticiens commencent à exercer à l'âge de 25 ans.

\section{Revenu}

La quatrième partie de notre questionnaire portait sur le revenu. La première observation que nous pouvons faire est que plus de la moitié des traductrices qui ont participé à notre sondage $(59 \%)$ ont un revenu qui se situe entre $20000 \$$ et $29999 \$$.

Nous avons également analysé la situation selon les différentes tranches d'âge. Près des trois quarts $(73 \%)$ de celles qui sont âgées de moins de 30 ans gagnent entre $20000 \$$ et 29999 . Plus de la moitié $(56 \%)$ de celles qui sont âgées de 30 à 39 ans gagnent également entre $20000 \$$ et $29999 \$$ tandis que les autres participantes dans cette catégorie d'âge gagnent soit entre $15000 \$$ et $19999 \$$, soit entre $30000 \$$ et $49999 \$$. Des participantes âgées de 40 à 49 ans, $46 \%$ gagnent entre $20000 \$$ et $29999 \$$, et un autre $46 \%$ gagnent entre $30000 \$$ et $49999 \$$. Donc, presque toutes celles qui ont entre 40 et 49 ans $(92 \%)$ gagnent plus de $20000 \$$. Nous pouvons conclure que la plupart des traductrices gagnent relativement bien leur vie, puisque $5 \%$ seulement ont un revenu annuel inférieur à $15000 \$$.

Nous aurions aimé pouvoir établir une comparaison entre le salaire des pigistes et celui des non-pigistes. Cela nous a toutefois été impossible puisque la majorité des pigistes consacrent moins d'heures à la traduction que les nonpigistes, qui travaillent à temps plein dans la plupart des cas. Il aurait fallu avoir une base commune, par exemple un salaire horaire ou hebdomadaire.

\section{Loisirs}

Cinquièmement, en analysant les données sur les loisirs, nous avons constaté que les traductrices semblent préférer les loisirs qui demandent une participation visuelle (elles ont surtout mentionné la lecture, le cinéma et le théâtre). La moitié des répondantes en ont fait leur premier choix, et $32 \%$ leur deuxième (voir tableau 6). Les traductrices sont aussi des sportives, puisqu'en effet les sports constituent le premier choix de $28 \%$ des répondantes et le deuxième choix de $29 \%$ d'entre elles.

\section{Degré de satisfaction}

Finalement, nous avons essayé de déterminer le degré de satisfaction au travail. Dans $75 \%$ des cas, les répondantes se sont déclarées satisfaites de leur situation professionnelle, tandis que près du quart d'entre elles envisageraient une autre carrière si elles avaient le loisir de recommencer. Il semble que l'insatisfaction augmente avec l'âge; $18 \%$ des répondantes de moins de 30 ans ne seraient pas traductrices, le pourcentage passant à $33 \%$ pour celles ayant entre 30 et 39 ans et à $56 \%$ pour celles ayant entre 40 et 49 ans. Nous avons pu également noter que plus du tiers (35\%) songent à s'orienter vers une autre carrière.

\section{Conclusion}

Les données que nous avons obtenues nous ont permis de tracer le profil général de la traductrice québécoise. Il ressort que la traductricetype a moins de 40 ans, est née au Québec, provient d'un milieu socio-économique élevé. Sa langue maternelle est le français et sa langue seconde l'anglais. Elle est probablement mariée, mais n'a pas nécessairement d'enfants. Elle possède un diplôme universitaire. Si elle est non-pigiste, elle travaille 37 heures par semaine dans le service de traduction d'une entreprise privée. En plus de ce travail, il ne lui arrive que très rarement de faire de la pige. Elle est présentement employée, et ses chances d'accéder à un poste de responsabilité sont aussi bonnes que celles de ses collègues masculins. Si elle est pigiste, elle travaille à temps partiel. C'est par goût qu'elle fait de la pige et elle trouve avantageux de choisir ses heures de travail, sa clientèle et les domaines de traduction. La traductrice-type traduit des textes commerciaux et techniques. La traduction est sa deuxième profession; auparavant, elle était secrétaire ou enseignante. Elle travaille dans ce domaine depuis environ 5 ans et gagne environ $25000 \$$ par année. Elle aime la lecture, le cinéma et le sport, qui occupent ses loisirs. Elle est satisfaite de son travail et n'hésiterait pas à recommencer.

\section{TABLEAUX}

Tableau 1 - Âge des traductrices

\begin{tabular}{lr}
\hline \multicolumn{1}{c}{ Âge } & \multicolumn{1}{c}{$\%$} \\
\hline moins de 30 ans & 23,0 \\
30 à 39 ans & 37,3 \\
40 à 49 ans & 23,0 \\
50 à 59 ans & 9,8 \\
60 à 69 ans & 4,7 \\
sans réponse & 2,2 \\
\hline TOTAL & 100,0 \\
\hline
\end{tabular}


Tableau 2 - Âge d'acquisition de la langue seconde

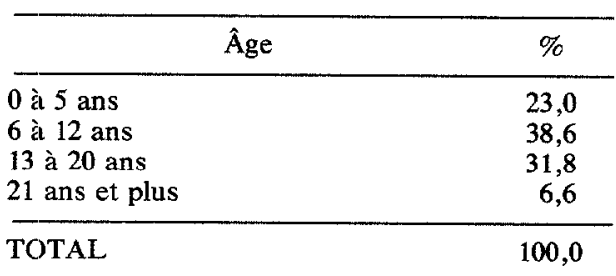

Tableau 3 - Moyen d'acquisition de la langue seconde

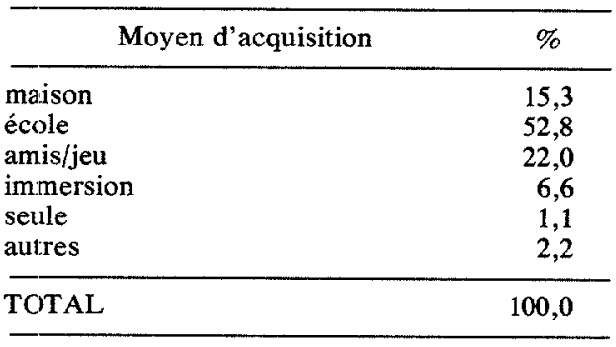

Tableau 4 - Proportion des hommes et des fernmes

\begin{tabular}{lccc}
\hline Nombre d'employés Cadres & Subalternes & Total \\
\hline & 151 & 231 & 382 \\
\cline { 2 - 4 } & $\%$ & $\%$ & $\%$ \\
Hommes & 29,1 & 23,4 & 25,7 \\
Femmes & 70,9 & 76,6 & 74,3 \\
\hline TCTAL & 100,0 & 100,0 & 100,0 \\
\hline
\end{tabular}

Tableau 5 - Domaine de spécialisation

\begin{tabular}{|c|c|}
\hline Domaine & $\%$ \\
\hline Technique & 16,5 \\
\hline Commercial & 30,8 \\
\hline Juridique & 5,5 \\
\hline Littéraire & 0,0 \\
\hline Médical & 5,5 \\
\hline Autres & 12,1 \\
\hline TO'TAL & 100,0 \\
\hline
\end{tabular}

Tableau 6 - Loisirs

\begin{tabular}{|c|c|c|}
\hline Genre d'occupation & 1er choix $\%$ & $2^{\mathrm{e}}$ choix $\%$ \\
\hline Manuel & 8,8 & 7,7 \\
\hline Auditif & 5,5 & 8,8 \\
\hline Visuel & 51,7 & 31,9 \\
\hline Sportif & 27,5 & 28,6 \\
\hline Autre & 6,5 & 13,2 \\
\hline $\mathrm{Ne}$ s'applique pas & 0,0 & 9,8 \\
\hline TOTAL & 100,0 & 100,0 \\
\hline
\end{tabular}

MrReIlle Boucher* JoHANNE THOMAS 